\title{
Recurrence of Thyroid Cancer: From Papillary to Anaplastic
}

\author{
Leah L. Awan ${ }^{\mathrm{a}, \mathrm{c}}$, Andrew A. Rosenthal ${ }^{\mathrm{b}}$, Eddy Carrillo ${ }^{\mathrm{b}}$, \\ Seong K. Lee ${ }^{\mathrm{b}}$, Rafael Sanchez ${ }^{\mathrm{b}}$, Chauniqua Kiffin ${ }^{\mathrm{b}}$
}

\begin{abstract}
Recurrent thyroid cancer often presents much more aggressively than the initial presentation. We present a case of a 50-year-old female, previously diagnosed and treated for papillary thyroid cancer, who subsequently developed a rapidly growing anaplastic thyroid cancer. This paper provides a discussion of anaplastic thyroid cancer in comparison to papillary cancer.
\end{abstract}

Keywords: Thyroid cancer; Anaplastic; Papillary

\section{Introduction}

Papillary thyroid cancer is often said to one of the "best" cancers to have, with respect to its low mortality and relative curability. However, on the other end of the spectrum is anaplastic thyroid cancer, an aggressive, invasive cancer, with almost certain mortality within a year. The transformation of thyroid cancer from a relatively "harmless" cancer to a fulminant insidious cancer is a rapid and dramatic change which can alter a patient's outcome.

\section{Case Report}

A 50-year-old white female presented with left sided solitary, palpable, hard, non-tender neck mass in the anterior triangle. She was found to have papillary thyroid carcinoma with regional lymph node metastasis status post total thyroidectomy

\footnotetext{
Manuscript accepted for publication April 25, 2013

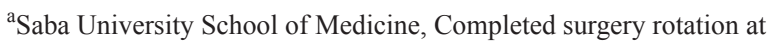
Memorial Regional Hospital, FL, USA

${ }^{\mathrm{b}}$ Memorial Regional Hospital, Hollywood, FL, USA

${ }^{\mathrm{c} C}$ Corresponding author: Leah L. Awan, Email: leahawan@gmail.com
}

doi: http://dx.doi.org/10.4021/jmc1267w in 2008. This was followed by high-dose I-131 radioactive iodine ablation therapy. In 2009 she had a total body I-131 scan that revealed no abnormalities.

Unfortunately, the patient was noncompliant with follow-up and thyroid lab testing. In March of 2012 the patient presented with a left sided neck mass. CT scan of the mass revealed a $3.1 \times 2.5 \times 4.0 \mathrm{~cm}$ hypervascular enhancing focus in the left neck, posterior to the left sternocleidomastoid muscle at the level of the thyroidectomy clips, which was new since period CT scan, suggesting recurrent disease.

The patient was advised to see a surgeon for further work up and treatment of the mass regretfully she was noncompliant until September 2012, when she presented to the emergency department with a painful, enlarging neck mass. Repeat CT revealed that the mass identified on the March 2012 CT had markedly increased in size to $8.5 \times 5.9 \mathrm{~cm}$ (Fig. 1). The lesion appeared to consist of intermediate soft tissue density with areas of high density suggestive of internal necrosis or hemorrhage. The lesion now extended to thoracic inlet and left supraclavicular region, rightwardly displacing the airway and laryngeal cartilage, and the left carotid, and left internal jugular vein appeared inseparable from the mass.

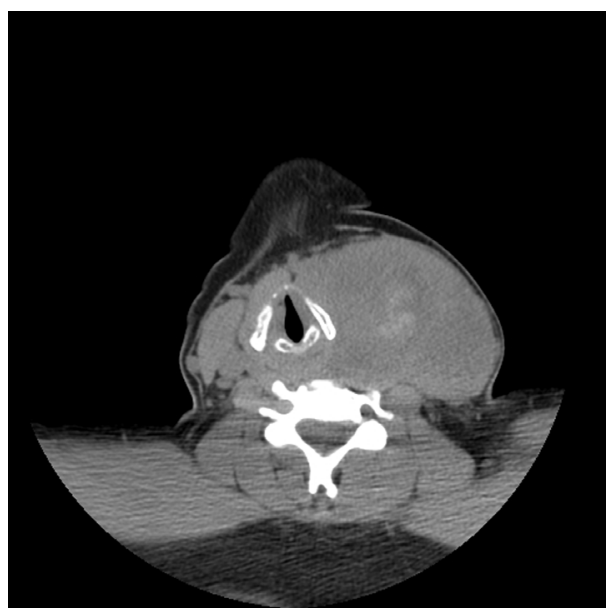

Figure 1. Neck computed topography without IV contrast reveals an extensive, heterogeneous infiltrative process in the left neck with areas of high density, suggestive of hemorrhage, and airway deviation to the right. 


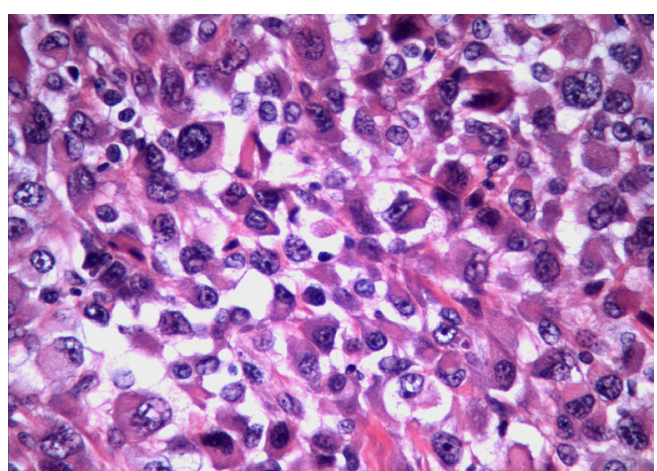

Figure 2. H\&E stained specimen of neck mass shows diffuse proliferation of highly pleomorphic, anaplastic cells. Abundant necrosis noted. Osteoclastic like giant cells present.

Her thyroglobin level at that time were greater than 1,000.

She underwent left radical neck dissection. Pathology revealed metastatic papillary thyroid carcinoma with multifocal anaplastic features (Fig. 2). The tumor was positive for extanodular extension, extensive skeletal muscle invasion, and prominent vascular invasion.

Postoperatively, the patient required thoracic duct repair and had wound dehiscence. The patient was discharged with plans for postoperative therapy once the wound closed. However, the patient was readmitted for tumor debulking and hemorrhage control. The tumor was too advanced for complete resection and involved the substernal and mediastinal components, crossing the midline, involving the ipsilateral brachial plexus and phrenic nerve. The tumor was very vascular, making debulking dangerous in terms of hemorrhage. The patient received of palliative chemotherapy and radiation therapy and underwent tracheostomy and gastrostomy, finally to leave the hospital in hospice care.

\section{Discussion}

Thyroid carcinoma accounts for about $1 \%$ of all cancers and makes up $0.2 \%$ of cancer deaths in the United States [1]. Thyroid cancers have been shown to be more prevalent in women than in men with a 3:1 ratio, and are usually diagnosed in the fourth to fifth decade of life. The most common presentation of thyroid cancers is an asymptomatic mass felt in the neck. Papillary is the most common form accounting for approximately $80 \%$, with anaplastic being very rare [1].

Papillary thyroid carcinoma is a well-differentiated thyroid carcinoma. It is the most common malignant tumor of the thyroid gland consisting of $80-85 \%$ of all thyroid malignancies, with a survival rate of $>95 \%$ at 5 years [1]. In contrast, anaplastic thyroid carcinoma is a poorly differentiated carcinoma. All anaplastic thyroid cancers are considered stage IV by TNM staging. Anaplastic thyroid carcinoma makes up about $1-2 \%$ of thyroid malignancies and accounts for $14-39 \%$ of thyroid cancer deaths, with a mean survival rate of 6 months from diagnosis, regardless of treatment [2].

Anaplastic cancer can arise denovo, from Hurthle cell variants of follicular carcinoma, or papillary thyroid cancer [2]. About $20 \%$ of patients with anaplastic carcinoma previously had a differentiated thyroid cancer, and 20 - 30\% have coexisting well-differentiated and anaplastic disease [3].

Histological transformation from well-differentiated thyroid carcinomas to poorly differentiated anaplastic carcinoma has been shown to result in very aggressive cancers [3]. BRAF mutation is often found in papillary thyroid carcinoma. Anaplastic carcinoma most commonly arises when the p53 tumor suppressor gene is lost [2].

Prognosis of anaplastic carcinoma is poor, regardless of therapy. Therapeutic options currently for anaplastic thyroid carcinoma are limited and have not proven effective. Surgical debulking of tumors followed by chemoradiotherapy is most affective against localized tumors. Unfortunately, anaplastic cancer often presents as more advanced, extracapular, metastatic disease. Doxorubicin and cisplatin have been the used for many years, but paclitaxel plus platinum compounds are also effective [4].

Deaths from asphyxia, constriction of the upper airway by the tumor, account for almost half of all anaplastic thyroid tumor related deaths. Surgery as a palliative measure followed by chemoradiotherapy can help reduce airway obstruction and improve survival by a few months $[4,5]$.

In conclusion, anaplastic thyroid carcinoma is a rare form of thyroid carcinoma with a poor prognosis, regardless of medical intervention. Current treatment options are ineffective in improving long-term survival. Further research is required to make progress with this unfortunately fatal disease.

\section{References}

1. LiVolsi VA. Papillary thyroid carcinoma: an update. Mod Pathol. 2011;24(Suppl 2):S1-9.

2. Taccaliti A, Silvetti F, Palmonella G, Boscaro M. Anaplastic thyroid carcinoma. Front Endocrinol (Lausanne). 2012;3:84.

3. Shiller SM, Konduri K, Harshman LK, Welch BJ, O'Brien JC. Recurrent thyroid cancer with changing histologic features. Proc (Bayl Univ Med Cent). 2010;23(3):304-310.

4. Perri F, Lorenzo GD, Scarpati GD, Buonerba C. Anaplastic thyroid carcinoma: A comprehensive review of current and future therapeutic options. World J Clin Oncol. 2011;2(3):150-157.

5. Goyal A, Gupta R, Mehmood S, Deo S, Mishra S, Bhatnagar S. Palliative and end of life care issues of carcinoma thyroid patient. Indian J Palliat Care. 2012;18(2):134137. 